\title{
IN SILICO AND PROTEOMICS APPROACHES SUGGEST UPREGULATION OF miR-146a-5p IN TNBC AND MODULATION OF CRITICAL PROTEINS
}

Tayana Schultz Jukoski', Talita Helen B. Gomig', Tamyres MIngorance Carvalho', Cicero Andrade Urban², Enilze Maria Souza Fonseca Ribeiro'

1 Universidade Federal do Paraná - Curitiba (PR), Brazil.

${ }^{2}$ Hospital Nossa Ssenhora das Graças - Curitiba (PR), Brazil.

Introduction: Breast cancer (BC) is the most common type of cancer after non-melanoma skin tumors among Brazilian women, with 61.61 cases estimated for 100 thousand women in 2020. New biomarkers, such as miRNAs and selected proteins, are essential in personalized medicine. Objectives: To evaluate the expression and possible role of miR-146a-5p in subtypes of BC. Methods: miRNAs selection was performed using in silico analysis from the TCGA (The Cancer Genome Atlas) database. Data from the miRNAs expression of 1,085 patients were accessed and compared among BC subtypes. After normalization, the Bayesian Student t-test evaluated differential expression (DE) analysis via the limma $\mathrm{R}$ package. Lists with DE miRNAs were divided between up and down-regulated status $(\mathrm{FC}= \pm 2)$. A second approach was to submit the data obtained from BC samples' mass spectrometry to IPA software to predict the activation/inhibition of upstream regulators in DE proteins lists in the tumor (T) versus contralateral tissue (CT). Results: A total of 206 upstream regulators were discovered at $\mathrm{p}<0.05 ; 12.6 \%$ of them were predicted with z-score values. In a TCGA analysis, miR-146a-5p was found up-regulated in triple-negative (TNBC) in comparison to other subtypes as a hormonal receptor (HR)+, HER2+, and non-TNBC (HR+ plus HER2+). The same was observed in TNBC cell lines by RT-qPCR. This miRNA was also predicted as an indirect regulator of CAT, LTF, CFH, and PGLYRP2 proteins in an IPA analysis. The proteomic analysis also demonstrated these molecules' relation with cancer hallmarks such as invasion, inflammation, and immune response. Conclusions: The results suggest that miR-146a-5p deregulation has a role in BC, mainly in TNBC, via the regulation of essential proteins. A better understanding of these molecules in $\mathrm{BC}$ is critical to define new biomarkers. 